Research Article

\title{
Discreteness and Convergence of Complex Hyperbolic Isometry Groups
}

\author{
Xi Fu \\ Department of Mathematics, Shaoxing University, Shaoxing, Zhejiang 312000, China \\ Correspondence should be addressed to Xi Fu; fuxi1984@hotmail.com
}

Received 10 June 2013; Accepted 2 October 2013

Academic Editor: Pedro M. Lima

Copyright (C) $2013 \mathrm{Xi} \mathrm{Fu}$. This is an open access article distributed under the Creative Commons Attribution License, which permits unrestricted use, distribution, and reproduction in any medium, provided the original work is properly cited.

We investigate the discreteness and convergence of complex isometry groups and some discreteness criteria and algebraic convergence theorems for subgroups of $\mathbf{P U}(n, 1)$ are obtained. All of the results are generalizations of the corresponding known ones.

\section{Introduction}

In 1976, Jørgensen obtained a very useful necessary condition for two-generator Kleinian groups of $M\left(\overline{\mathbb{R}}^{2}\right)$, which is known as Jørgensen's inequality. As an application, he obtained the following $[1,2]$.

Theorem J. A nonelementary subgroup $G$ of $M\left(\overline{\mathbb{R}}^{2}\right)$ is discrete if and only if each two-generator subgroup in $G$ is discrete.

Furthermore, Gilman [3] and Isachenko [4] showed that the discreteness of all two-generator subgroups of $G$, where each generator is loxodromic, is enough to secure the discreteness of G. See [5-8] and so forth for some other discussions along this line.

It is interesting to generalize Theorem J into the higher dimensional case. By adding some conditions, several generalizations of Theorem J into $M\left(\overline{\mathbb{R}}^{n}\right)(n \geq 3)$ have been obtained; see [9-13] and so forth. In 2005, Wang et al. [14] proved the following.

Theorem WLC. Let $G \subset M\left(\overline{\mathbb{R}}^{n}\right)$ be nonelementary and $f \in G$ loxodromic. Then $G$ is discrete if and only if $W Y(G)$ is discrete and each nonelementary subgroup $\left\langle f, \mathrm{gfg}^{-1}\right\rangle$ is discrete, where $g \in G$.

Here

$$
W Y(G)=\left\{h:\left.h\right|_{M(G)}=I, h \in G\right\}
$$

and $M(G)$ is the smallest $G$-invariant hyperbolic subspace whose boundary contains the limit set $L(G)$ of $G$ (cf. [15]).

Since the real hyperbolic plane can be viewed as a complex hyperbolic 1-space $\mathbb{H}_{\mathbb{C}}^{1}$, it is natural to generalize these results mentioned above to the setting of complex hyperbolic space. Recently, Qin and Jiang [16] proved the following.

Theorem QJ 1. Let $G$ be an $n$-dimensional subgroup of $\mathbf{P U}(n, 1)$ and $f$ a nonelliptic element in $\mathbf{P U}(n, 1)$. If for each loxodromic (resp., regular elliptic) element $g \in G$ the twogenerator group $\langle f, g\rangle$ is discrete, then $G$ is discrete.

Theorem QJ 2. Let $G$ be an n-dimensional subgroup of $\mathbf{P U}(n, 1)$ and $f$ a regular elliptic element in $\mathbf{P U}(n, 1)$. If for each loxodromic (resp., regular elliptic) element $g \in G$ the twogenerator group $\langle f, g\rangle$ is discrete, then $G$ is discrete.

Here $G$ is called $n$-dimensional if it does not leave a point in $\partial \mathbb{C}_{\mathbb{C}}^{n}$ or a proper totally geodesic submanifold of $\mathbb{G} \mathbb{C}_{\mathbb{C}}^{n}$ invariant. Obviously, if $G$ is $n$-dimensional, then $G$ is nonelementary and $M(G)=\mathbb{T}_{\mathbb{C}}^{n}$.

Motivated by Theorem WLC, a natural question will be asked: can we use the discreteness of subgroups $\left\langle f, g f g^{-1}\right\rangle$ to determine the discreteness of $G$ in Theorems QJ1 and QJ2? In this paper, we will give this question a positive answer (see Section 3).

Let $\mathbb{G}$ be the Möbius group $M\left(\overline{\mathbb{R}}^{n}\right)$ or the complex hyperbolic isometry group $\mathbf{P U}(n, 1)$. 
Definition 1. Let $\left\{G_{r, i}\right\}$ be a sequence of subgroups in $\mathbb{G}$ and each $G_{r, i}$ be generated by $g_{1, i}, g_{2, i}, \ldots, g_{r, i}$. If, for each $t \in$ $\{1,2, \ldots, r\}(r<\infty)$,

$$
g_{t, i} \longrightarrow g_{t} \in \mathbb{G} \quad \text { as } i \longrightarrow \infty
$$

then we say that $\left\{G_{r, i}\right\}$ converges algebraically to $G_{r}=\left\langle g_{1}\right.$, $\left.g_{2}, \ldots, g_{r}\right\rangle$ and $G_{r}$ is called the algebraic limit group of $\left\{G_{r, i}\right\}$. If for each $i, G_{r, i}$ is a Kleinian group, then the question when $G_{r}$ is still a Kleinian group has attracted much attention. Jørgensen and Klein proved that $G_{r}$ is still a Kleinian group, when $n=2$. For the higher dimensional case, there are a number of discussions; see $[11,12,17]$.

When $\mathbb{G}=\mathbf{P U}(n, 1)$, Cao proved [18] the following.

Theorem C 1. Let $\left\{G_{r, i}\right\}$ be a sequence of groups of $\mathbb{G}$. If each $G_{r, i}$ is discrete, then the algebraic limit group $G_{r}$ of $\left\{G_{r, i}\right\}$ is either a complex Kleinian group, or it is elementary, or $W(G r)$ is not finite.

Theorem C 2. Let $G_{r}$ be the algebraic limit group of complex Kleinian groups $\left\{G_{r, i}\right\}$ of $\mathbb{G}$. If $\left\{G_{r, i}\right\}$ satisfies IP-condition, then $G_{r}$ is a complex Kleinian group.

Here $\left\{G_{r, i}\right\}$ satisfies IP-condition means that $\left\{G_{r, i}\right\}$ satisfies the following conditions: for any sequence $\left\{f_{i}\right\}, f_{i} \in G_{r, i}$, if $\operatorname{card}\left[\operatorname{fix}\left(f_{i}\right)\right]=\infty$ for each $i$, and $f_{i} \rightarrow f$ as $i \rightarrow \infty$ with $f$ being the identity or parabolic, then $\left\{f_{i}\right\}$ has uniformly bounded torsion (see [18]).

In this paper, we will discuss the discreteness criteria and algebraic convergence theorems for subgroups of $\mathbf{P U}(n, 1)$ further. The rest of this paper is organized as follows: in Section 2, we introduce some preliminary results that we need in the sequel; in Section 3, we show three discreteness criteria for subgroups of $\mathbf{P U}(n, 1)$; finally Section 4 is dedicated to three algebraic convergence theorems for complex Kleinian groups.

\section{Preliminaries}

Let $\mathbb{C}^{n, 1}$ be the complex vector space of dimension $n+1$ with the Hermitian form

$$
\langle z, w\rangle=z_{1} \bar{w}_{1}+z_{2} \bar{w}_{2}+\cdots+z_{n} \bar{w}_{n}-z_{n+1} \bar{w}_{n+1},
$$

where $z, w$ are the column vectors in $\mathbb{C}^{n+1}$. Consider the following subspaces of $\mathbb{C}^{n, 1}$ :

$$
\begin{gathered}
V_{0}=\left\{z \in \mathbb{C}^{n, 1}-\{0\}:\langle z, z\rangle=0\right\}, \\
V_{-}=\left\{z \in \mathbb{C}^{n, 1}:\langle z, z\rangle<0\right\} .
\end{gathered}
$$

Let $P: \mathbb{C}^{n+1}-\{0\} \rightarrow \mathbb{C} \mathbb{P}^{n}$ be the canonical projection from $\mathbb{C}^{n+1}-\{0\}$ onto the complex hyperbolic space $\mathbb{C P}^{n}$. The complex hyperbolic space is defined to be $\mathbb{U}_{\mathbb{C}}^{n}=P V_{-}$and $\partial \mathbb{U}_{\mathbb{C}}^{n}=P V_{0}$ is its boundary. The biholomorphic isometry group of $\mathbb{U}_{\mathbb{C}}^{n}$ is given by the projective unitary group $\mathbf{P U}(n, 1)$. For a nontrivial element $g$ of $\mathbf{P U}(n, 1)$, we say that $g$ is elliptic if it has a fixed point in $\mathbb{H}_{\mathbb{C}}^{n}, g$ is parabolic if it has only one fixed point in $\partial \mathbb{C}_{\mathbb{C}}^{n}$, and $g$ is loxodromic if it has exactly two different fixed points in $\partial \mathbb{U}_{\mathbb{C}}^{n}$.

For elliptic element $g \in \mathbf{P U}(n, 1)$, let $\Lambda_{0}$ and $\Lambda_{i}$ $(i=1,2, \ldots, n)$ be its negative and positive eigenvalues, respectively. Then the fixed point set of $g$ in $\mathbb{T}_{\mathbb{C}}^{n}$ contains only one point if $\Lambda_{0} \neq \Lambda_{i}$ and is a totally geodesic submanifold, which is equivalent to $\mathbb{U}_{\mathbb{C}}^{m}$ if $\Lambda_{0}$ coincides with exact $m$ of class $\Lambda_{i}(m<n)$. We call $g$ regular elliptic if $\Lambda_{s} \neq \Lambda_{t}$, where $s, t \in$ $\{0,1, \ldots, n\}$ and $s \neq t$. Obviously, if $g$ is regular elliptic, then $g$ has only one fixed point in $\mathbb{H}_{\mathbb{C}}^{n}$. The following proposition follows directly from [19].

Proposition 2. The regular elliptic (resp., loxodromic) elements of $\mathbf{P U}(n, 1)$ form an open set.

Let $G$ be a subgroup of $\mathbf{P U}(n, 1)$. The limit set $L(G)$ of $G$ is defined as

$$
L(G)=\overline{G(p)} \cap \partial \mathbb{T}_{\mathbb{C}}^{n}, \quad p \in \mathbb{H}_{\mathbb{C}}^{n}
$$

$G$ is called nonelementary if $L(G)$ contains more than two points; otherwise, it is called elementary. We call a subgroup $G$ of $\mathbf{P U}(n, 1)$ complex Kleinian group if it is discrete and nonelementary. For a nonelementary subgroup $G$ of $\mathbf{P U}(n, 1)$, we denote by $M(G)$ the smallest totally geodesic submanifold of $G$ whose boundary contains the limit set $L(G)$. It is easy to see that $M(G)$ is $G$-invariant since $L(G)$ is $G$-invariant. As in [18], the subgroup $W(G)$ of $G$ is defined as

$$
W(G)=\left\{g:\left.g\right|_{M(G)}=I, g \in G\right\} .
$$

For an element $f \in \mathbf{P U}(n, 1)$, we denote $N(f)=\|f-I\|$, where $\|\cdot\|$ is the Hilbert-Schmidt norm. Then we have the following.

Lemma 3 (see $[18,20]$ ). Suppose that two elements $f, g \in$ $\mathrm{PU}(n, 1)$ generate a complex Kleinian group.

(1) If $f$ is parabolic or loxodromic, then

$$
\max \{N(f), N([f, g])\} \geq 2-\sqrt{3},
$$

where $[f, g]=f g f^{-1} g^{-1}$ is the commutator of $f$ and $g$.

(2) If $f$ is elliptic, then

$$
\max \left\{N(f), N\left(\left[f, g^{q}\right]\right): q=1,2,3, \ldots, n+1\right\} \geq 2-\sqrt{3} .
$$

\section{Discreteness Criteria}

In this section, we prove the following theorems.

Theorem 4. Let $G$ be an $n$-dimensional subgroup of $\mathbf{P U}(n, 1)$ and $f$ a nonelliptic element in $\mathrm{PU}(n, 1)$. If for each loxodromic (resp., regular elliptic) element $g \in G$ the two-generator group $\left\langle f, \mathrm{gfg}^{-1}\right\rangle$ is discrete, then $G$ is discrete.

Theorem 5. Let $G$ be an $n$-dimensional subgroup of $\mathbf{P U}(n, 1)$ and $f$ a regular elliptic element with finite order $k(k \geq 3)$ 
in $\mathbf{P U}(n, 1)$. If for each loxodromic (resp., regular elliptic) element $g \in G$ the two-generator group $\left\langle f, g f g^{-1}\right\rangle$ is discrete, then $G$ is discrete.

When $f$ is elliptic (may not be regular), we have the following.

Theorem 6. Let $G$ be an $n$-dimensional subgroup of $\mathbf{P U}(n, 1)$ and $f$ an elliptic element with finite order $k(k \geq 3)$ in $\mathbf{P U}(n, 1)$. If, for each loxodromic (resp., regular elliptic) element $g \in G$ the two-generator group $\langle f, g\rangle$ is discrete, then $G$ is discrete.

In order to prove the above theorems, we need the following lemma which is a classification of elementary subgroups of $\mathbf{P} \mathbf{U}(n, 1)$.

Lemma 7. Let $G$ be a subgroup of $\mathbf{P U}(n, 1)$.

(1) If $G$ contains a loxodromic element, then $G$ is elementary if and only if it fixes a point in $\partial \mathbb{G}_{\mathbb{C}}^{n}$ or a point-pair $\{x, y\}$ in $\partial \mathbb{Q}_{\mathbb{C}}^{n}$.

(2) If $G$ contains a parabolic element but no loxodromic element, then $G$ is elementary if and only if it fixes a point in $\partial \square \square_{\mathbb{C}}^{n}$.

(3) If $G$ is purely elliptic, then G fixes a point in $\overline{\mathbb{Z}}_{\mathbb{C}}^{n}$.

Proof of Theorem 4. Firstly, we prove the case when each $g$ is loxodromic. Suppose not. Then $G$ is dense in $\mathbf{P U}(n, 1)$ according to Corollary 4.5.1 of [15]. By Proposition 2, there exists a sequence $\left\{g_{i}\right\}$ in $G$ such that each $g_{i}$ is loxodromic and $g_{i} \rightarrow I$ as $i \rightarrow \infty$. Then, for large enough $i$, we have

$$
N\left(g_{i} f g_{i}^{-1} f^{-1}\right)+\sum_{q=1}^{n+1} N\left(\left[g_{i} f g_{i}^{-1} f^{-1}, f^{q}\right]\right)<2-\sqrt{3} .
$$

Since $f$ is nonelliptic and $\left\langle f, g_{i} f g_{i}^{-1} f^{-1}\right\rangle=\left\langle f, g_{i} f g_{i}^{-1}\right\rangle$, by Lemma 3, we know that, for all large enough $i,\left\langle f, g_{i} f g_{i}^{-1}\right\rangle$ are elementary. This implies that

$$
\operatorname{fix}(f) \cap \operatorname{fix}\left(g_{i}\right) \neq \emptyset .
$$

Since $G$ is nonelementary, we can find three loxodromic elements $h_{s}(s=1,2,3)$ in $G$ such that

$$
\text { fix }(f) \cap \operatorname{fix}\left(h_{s}\right)=\emptyset, \quad \text { fix }\left(h_{j}\right) \cap \operatorname{fix}\left(h_{k}\right)=\emptyset,
$$

where $i, k \in\{1,2,3\}$ and $j \neq k$. It follows from a discussion similar to the above that we can obtain that, for large enough $i$,

$$
\text { fix }(f) \cap \operatorname{fix}\left(h_{s} g_{i} h_{s}^{-1}\right) \neq \emptyset, \quad s=1,2,3 .
$$

Since $f$ is nonelliptic, that is, $\operatorname{fix}(f)$ contains less than three points; it is a contradiction.

Now, we come to prove the case when each $g$ is regular elliptic. Suppose that $G$ is nondiscrete. Similarly, by Proposition 2, we can find a sequence $\left\{g_{i}\right\}$ in $G$ such that each $g_{i}$ is regular elliptic and $g_{i} \rightarrow I$ as $i \rightarrow \infty$. This implies that, for sufficiently large $i$, the subgroups $\left\langle f, g_{i} f g_{i}^{-1}\right\rangle$ are elementary. It follows that

$$
\text { fix }(f)=\operatorname{fix}\left(g_{i}\right) .
$$

It is a contradiction since $f$ is nonelliptic and $g_{i}$ is regular elliptic.

This completes the proof.

Proof of Theorem 5. The proof of Theorem 5 follows from a discussion similar to that in the proof of Theorem 4.

Proof of Theorem 6. We only prove the case when $g$ is loxodromic; similar arguments can be applied to the case when $g$ is regular elliptic. Suppose that $G$ is nondiscrete. Then there exists a sequence $\left\{g_{i}\right\} \subset G$ such that, for each $i, g_{i}$ is loxodromic and

$$
g_{i} \longrightarrow I \quad \text { as } i \longrightarrow \infty
$$

Since $G$ is $n$-dimensional, we can find finitely many loxodromic elements $h_{1}, h_{2}, \ldots, h_{t}$ in $G$ such that the set $S=$ $\left\{A_{\text {fix }\left(h_{1}\right)}, A_{\text {fix }\left(h_{2}\right)}, \ldots, A_{\text {fix }\left(h_{t}\right)}\right\}$ can span the whole complex hyperbolic space $\mathbb{U}_{\mathbb{C}}^{n}$, where $A_{\text {fix }(h)}$ is the attractive fixed point of $h$. For each $k(k=1,2, \ldots, t)$, let $U_{A_{\mathrm{fx}}\left(h_{k}\right)}$ be a small neighbourhood of $A_{\text {fix }\left(h_{k}\right)}$ in $\overline{\mathbb{M}}_{\mathbb{C}}^{n}$; then there exists an integer $N$ such that

$$
h_{k}^{N}(\operatorname{fix}(f)) \subset U_{A_{\text {fix }\left(h_{k}\right)}} .
$$

Since

$$
\begin{gathered}
\left\langle h_{k}^{N} f h_{k}^{-N}, g_{i}\right\rangle=h_{k}^{N}\left\langle f, h_{k}^{-N} g_{i} h_{k}^{N}\right\rangle h_{k}^{-N}, \\
\max \left\{N\left(h_{k}^{-N} g_{i} h_{k}^{N}\right), N\left(\left[h_{k}^{-N} g_{i} h_{k}^{N}, f\right]\right)\right\}<2-\sqrt{3},
\end{gathered}
$$

for large enough $i$, we can see that the subgroups $\left\langle h_{k}^{N} f^{2} h_{k}^{-N}, g_{i}\right\rangle$ are elementary. By Lemma 7, we know that, for each $k,(k=1,2, \ldots, t)$,

$$
\text { fix }\left(g_{i}\right) \cap U_{A_{\text {fix }\left(h_{k}\right)}} \neq \emptyset .
$$

Obviously, it is a contradiction.

\section{Algebraic Convergence}

In this section, we discuss the algebraic convergence of complex hyperbolic Kleinian groups. Firstly, we generalize Theorem $\mathrm{Cl}$ into the following form.

Theorem 8. Let $\left\{G_{r, i}\right\}$ be a sequence of groups of $\mathbf{P U}(n, 1)$ and $G_{r}$ be its algebraic limit group. Then we have the following.

(1) If, for each $i, G_{r, i}$ is a complex Kleinian group, then $G_{r}$ is nonelementary and $G_{r}$ is discrete if and only if each one-generator subgroup of $W\left(G_{r}\right)$ is discrete.

(2) If, for each $i, G_{r, i}$ is discrete, then $G_{r}$ is elementary if and only if for large enough $i$, all $G_{r, i}$ are elementary. 
Proof. The proof of (1). The nonelementariness of $G_{r}$ follows from [21, Theorem 1.4]. Now, we come to prove that if $G_{r}$ is nondiscrete, then there is an element $f \in W\left(G_{r}\right)$ such that the subgroup $\langle f\rangle$ is nondiscrete. Suppose that $G_{r}$ is nondiscrete. Since $r<\infty$ (that is, $G_{r}$ is finitely generated), by Selberg's Lemma we know that $G_{r}$ contains a torsion free subgroup $G_{r_{1}}$ with finite index which is nonelementary and nondiscrete either. Then there exists a sequence $\left\{f_{j}\right\}$ in $G_{r_{1}}$ such that

$$
f_{j} \longrightarrow I \quad \text { as } j \longrightarrow \infty \text {. }
$$

As $G_{r_{1}}$ is nonelementary, we can find finitely many loxodromic elements $g_{1}, g_{2}, \ldots, g_{k}$ in $G_{r_{1}}$ such that the set $\left\{\right.$ fix $\left(g_{1}\right)$, fix $\left.\left(g_{2}\right), \ldots, f i x\left(g_{k}\right)\right\}$ spans $\partial M\left(G_{r_{1}}\right)$, the boundary of $M\left(G_{r_{1}}\right)$. Then, for large enough $j$, we have

$$
N\left(f_{j}\right)+\sum_{q=1}^{n+1} N\left(\left[f_{j}, g_{s}^{q}\right]\right)<2-\sqrt{3}, \quad s \in\{1,2, \ldots, k\} .
$$

Let $f_{i, j}$ and $g_{i, s}$ be the corresponding elements of $f_{j}$ and $g_{s}$ in $G_{r, i}$, respectively. Then, for large enough $i$ and $j$,

$$
N\left(f_{i, j}\right)+\sum_{q=1}^{n+1} N\left(\left[f_{i, j}, g_{i, s}^{q}\right]\right)<2-\sqrt{3}
$$

Lemma 3 implies that, for large enough $i$ and $j$, the subgroups $\left\langle f_{i, j}, g_{i, s}\right\rangle$ are elementary. Since the loxodromic elements of PU $(n, 1)$ form an open set, we know that, for sufficiently large $i, g_{i, s}$ are loxodromic as well. It follows that

$$
\operatorname{fix}\left(g_{i, s}\right) \subset \operatorname{fix}\left(f_{i, j}\right),
$$

which shows that, for $s \in\{1,2, \ldots, k\}$ and all sufficiently large $j$,

$$
\operatorname{fix}\left(g_{s}\right) \subset \operatorname{fix}\left(f_{j}\right) .
$$

Thus, for all sufficiently large $j$,

$$
f_{j} \in W\left(G_{r_{1}}\right) \text {. }
$$

Since $G_{r_{r}}$ is torsion free, we know that there exists an element $f \in W\left(G_{r_{1}}\right)$ such that $\langle f\rangle$ is nondiscrete. Note that $M\left(G_{r}\right)=$ $M\left(G_{r_{1}}\right)$, so $f \in W\left(G_{r}\right)$. Hence, the conclusion of (1) follows.

The proof of (2). We only need to prove that if, for large enough $i$, all $G_{r, i}$ are elementary, then is $G_{r}$ since the converse is trivial by (1). Suppose that $G_{r}$ is nonelementary. Then we can find two loxodromic elements $f$ and $g$ in $G_{r}$ such that

$$
\operatorname{fix}(f) \cap \operatorname{fix}(g)=\emptyset .
$$

Let $f_{i}$ and $g_{i}$ be the corresponding elements of $f$ and $g$ in $G_{r, i}$, respectively. Then, for large enough $i$, we have

$$
\text { fix }\left(f_{i}\right) \cap \text { fix }\left(g_{i}\right)=\emptyset .
$$

It follows a discussion similar to that in the proof of (1) that, for large enough $i$, both $f_{i}$ and $g_{i}$ are loxodromic. This shows that, for large enough $i$, all $G_{r, i}$ are nonelementary. It is a contradiction.
Definition 9. Let $\left\{G_{i}\right\}$ be a sequence of complex Kleinian groups of $\mathbf{P U}(n, 1)$. We say that $\left\{G_{i}\right\}$ satisfies $E$-condition if there is no sequence $\left\{f_{i}\right\}, f_{i} \in W\left(G_{i}\right)$ such that $f_{i} \rightarrow f$ as $i \rightarrow \infty$, where $f$ is an elliptic element with infinite order.

In the following, we give an example which shows that, if the sequence $\left\{G_{i}\right\}$ does not satisfy IP-condition but $E$ condition, then the limit group $G_{r}$ is still a complex Kleinian groups.

Example 10. Suppose that $H$ is a purely loxodromic nonelementary subgroup of $\mathbf{P U}(1,1)$ and, for each $j$,

$$
f_{j}=\left[\begin{array}{ccc}
e^{i\left(1 / 2^{j}\right)} & 0 & 0 \\
0 & 1 & 0 \\
0 & 0 & 1
\end{array}\right]
$$

Let $\widetilde{H}$ be the Poincaré extension of $H$ in $\mathbf{P U}(2,1)$ and $G_{j}=$ $\left\langle\widetilde{H}, f_{j}\right\rangle$. Then it is easy to see that the algebraic limit group $G$ of $\left\{G_{j}\right\}$ is a complex Kleinian group. Note that $f_{j} \rightarrow I$ as $j \rightarrow \infty$; we know that $\left\{G_{j}\right\}$ does not satisfy IP-condition but E-condition.

As applications of Theorem 8 and E-condition, we have the following.

Theorem 11. Let $G_{r}$ be the algebraic limit group of complex Kleinian groups $\left\{G_{r, i}\right\}$ of $\mathbf{P U}(n, 1)$. If $\left\{G_{r, i}\right\}$ satisfies $E$ condition, then $G_{r}$ is a complex Kleinian group.

Proof. By Theorem 8(1), we know that $G_{r}$ is nonelementary. Suppose that $G_{r}$ is nondiscrete. Then there exist an elliptic element $f \in W\left(G_{r}\right)$ and an integer sequence $\left\{n_{j}\right\}$ such that $\operatorname{ord}(f)=\infty$ and

$$
f^{n_{j}} \longrightarrow I \quad \text { as } n_{j} \longrightarrow \infty
$$

For each $n_{j}$, let $f_{i}^{n_{j}}$ be the corresponding element of $f^{n_{j}}$ in $G_{r, i}$. By [21, Lemma 4.2], we know that $f_{i}^{n_{j}} \in W\left(G_{r, i}\right)$. It follows from the hypothesis that $\left\{G_{r, i}\right\}$ satisfies $E$-condition; we have $f_{i}^{n_{j}}=I$ for large enough $i$. This implies that $f^{n_{j}}=I$. It is a contradiction.

The proof is completed.

When $r \leq \infty$, Wang [17] proved the following.

Theorem W. Let $r \leq \infty$. If the generator system $\left\{g_{t, i}\right\}_{t=1}^{r}$ of $G_{r, i}$ satisfies that none are elliptic and no two have any fixed point in common, and, if all $G_{r, i}$ are Kleinian groups, then

(1) all the generators $g_{t}=\lim _{i \rightarrow \infty} g_{t, i}$ are neither elliptic nor identity;

(2) if $G_{r}=\left\langle g_{1}, g_{2}, \ldots, g_{r}\right\rangle$ is nonelementary and $W\left(G_{r}\right)$ is discrete, then $G_{r}$ is discrete.

It easily follows a similar argument as in the proof of Theorem 8 and we can obtain the following.

Theorem 12. Let $r \leq \infty$. If the generator system $\left\{g_{t, i}\right\}_{t=1}^{r}$ of $G_{r, i}$ satisfies that none are elliptic and no two have any fixed point in common, and, if all $G_{r, i}$ are discrete, then 
(1) $G_{r}=\left\langle g_{1}, g_{2}, \ldots, g_{r}\right\rangle$ is nonelementary;

(2) $G_{r}$ is discrete if and only if $W\left(G_{r}\right)$ is discrete.

\section{Acknowledgment}

The research was partly supported by Tian-Yuan Foundation (no. 11226096).

\section{References}

[1] T. Jørgensen, "On discrete groups of Möbius transformation," American Journal of Mathematics, vol. 98, pp. 739-749, 1976.

[2] T. Jørgensen, “A note on subgroups of $S L(2, \mathbb{C}), "$ The Quarterly Journal of Mathematics, vol. 28, no. 110, pp. 209-211, 1977.

[3] J. Gilman, "Inequalities in discrete subgroups of $\operatorname{PSL}(2, \mathbb{R})$," Canadian Journal of Mathematics, vol. 40, no. 1, pp. 115-130, 1988.

[4] N. A. Isachenko, "Systems of generators of subgroups of $\operatorname{PSL}(2, \mathbb{C})$," Siberian Mathematical Journal, vol. 31, no. 1, pp. 162$165,1990$.

[5] A. F. Beardon, The Geometry of Discrete Groups, vol. 91 of Graduate Texts in Mathematics, Springer, New York, NY, USA, 1983.

[6] P. Tukia and X. Wang, "Discreteness of subgroups of $S L(2, \mathbb{C})$ containing elliptic elements," Mathematica Scandinavica, vol. 91, no. 2, pp. 214-220, 2002.

[7] X. Wang and W. Yang, "Discreteness criterions for subgroups in $S L(2, \mathbb{C})$," Mathematical Proceedings of the Cambridge Philosophical Society, vol. 124, no. 1, pp. 51-55, 1998.

[8] S. Yang, "Test maps and discrete groups in $S L(2, \mathbb{C})$," Osaka Journal of Mathematics, vol. 46, no. 2, pp. 403-409, 2009.

[9] W. Abikoff and A. Haas, "Nondiscrete groups of hyperbolic motions," The Bulletin of the London Mathematical Society, vol. 22, no. 3, pp. 233-238, 1990.

[10] M. Chen, "Discreteness and convergence of Möbius groups," Geometriae Dedicata, vol. 104, pp. 61-69, 2004.

[11] A. Fang and B. Nai, "On the discreteness and convergence in $n$-dimensional Möbius groups," Journal of the London Mathematical Society, vol. 61, no. 3, pp. 761-773, 2000.

[12] G. J. Martin, “On discrete Möbius groups in all dimensions: a generalization of Jørgensen's inequality," Acta Mathematica, vol. 163, no. 3-4, pp. 253-289, 1989.

[13] X. Wang and W. Yang, "Discreteness criteria of Möbius groups of high dimensions and convergence theorems of Kleinian groups," Advances in Mathematics, vol. 159, no. 1, pp. 68-82, 2001.

[14] X. Wang, L. Li, and W. Cao, "Discreteness criteria for Möbius groups acting on $\overline{\mathbb{R}^{n}}$," Israel Journal of Mathematics, vol. 150, pp. 357-368, 2005.

[15] S. S. Chen and L. Greenberg, "Hyperbolic spaces," in Contributions to Analysis a Collection of Papers Dedicated to Lipman Bers, pp. 49-87, Academic Press, New York, NY, USA, 1974.

[16] H. Qin and Y. Jiang, "Discreteness criteria based on a test map in PU(n,1)," Proceedings-Mathematical Sciences, vol. 122, no. 4, pp. 519-524, 2012.

[17] X. Wang, "Algebraic convergence theorems of $n$-dimensional Kleinian groups," Israel Journal of Mathematics, vol. 162, pp. 221233, 2007.
[18] W. Cao, "Algebraic convergence theorems of complex Kleinian groups," Glasgow Mathematical Journal, vol. 55, no. 1, pp. 1-8, 2013.

[19] W. M. Goldman, Complex Hyperbolic Geometry, Oxford University Press, Oxford, UK, 1999.

[20] B. Dai, A. Fang, and B. Nai, "Discreteness criteria for subgroups in complex hyperbolic space," Proceedings of the Japan Academy, vol. 77, no. 10, pp. 168-172, 2001.

[21] M. Kapovich, "On sequences of finitely generated discrete groups," in In the Tradition of Ahlfors-Bers. V, vol. 510 of Contemporary Mathematics, pp. 165-184, American Mathematical Society, Providence, RI, USA, 2010. 


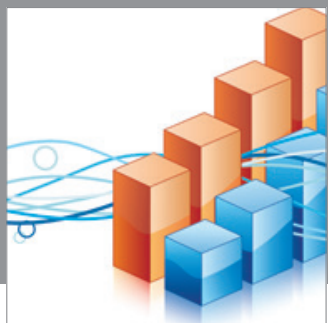

Advances in

Operations Research

mansans

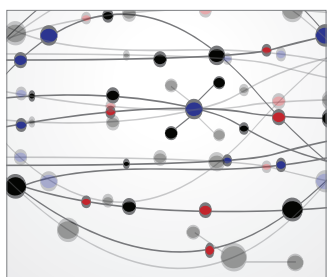

The Scientific World Journal
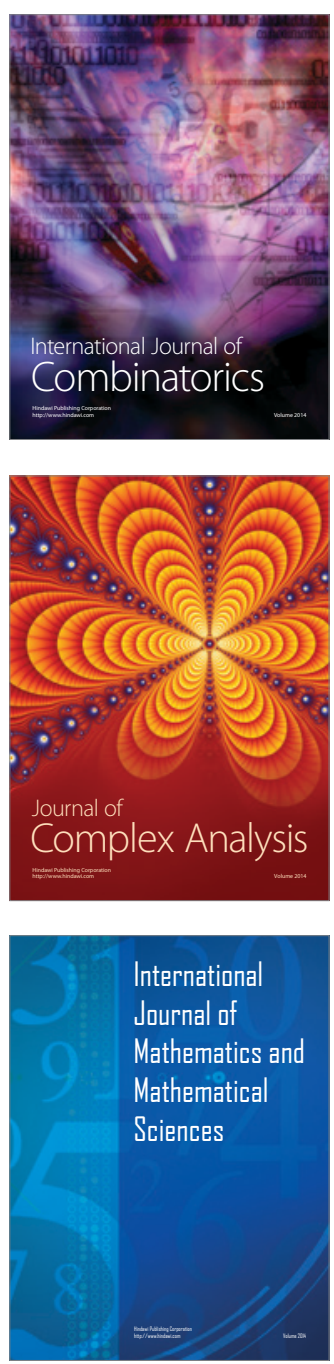
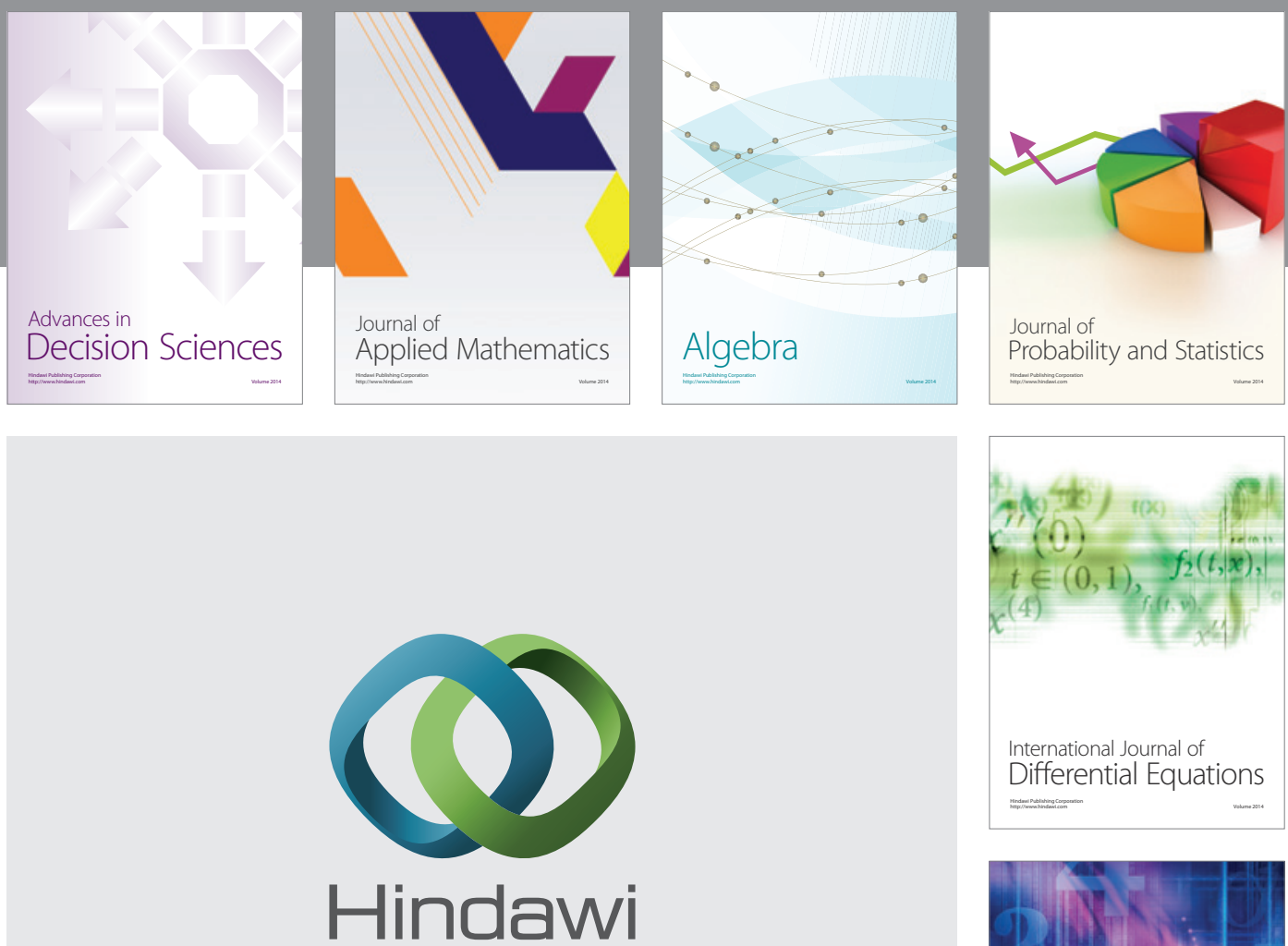

Submit your manuscripts at http://www.hindawi.com
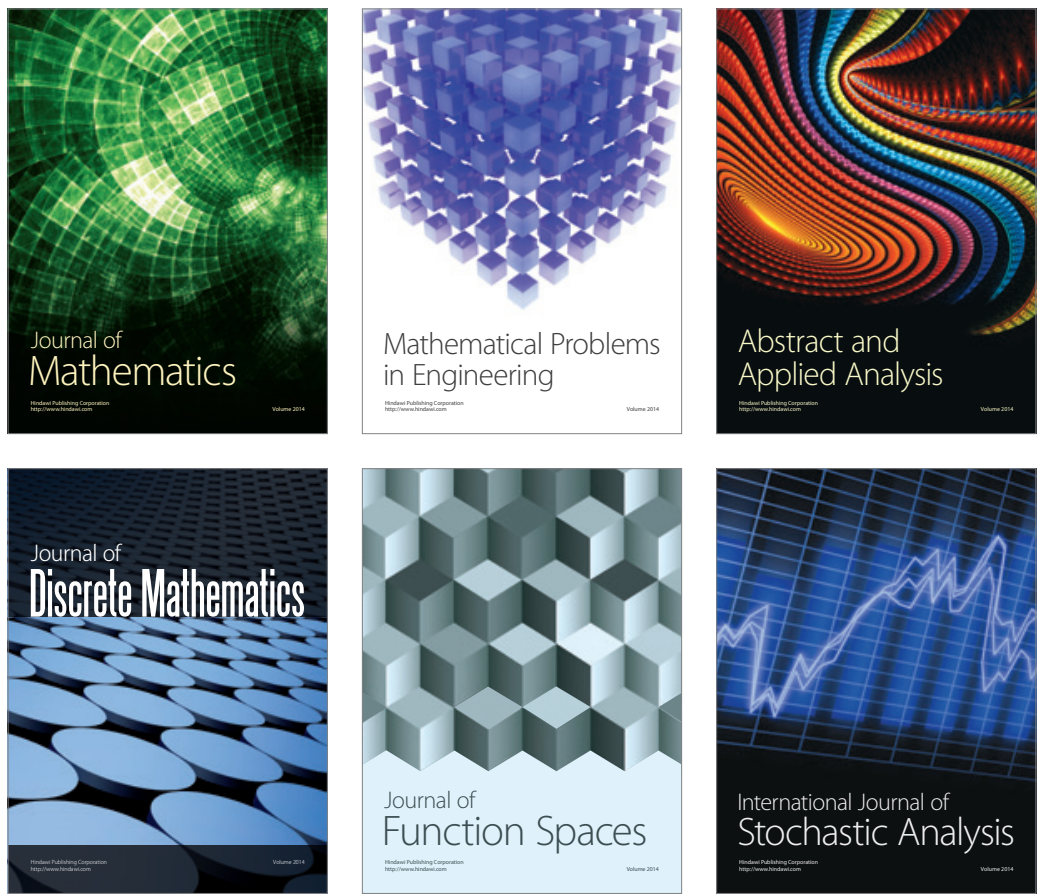

Journal of

Function Spaces

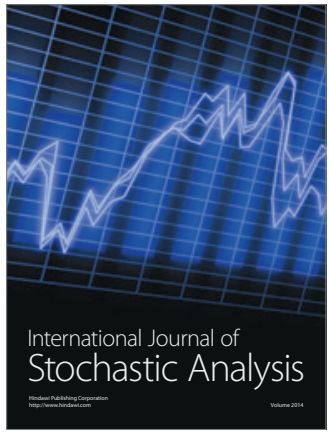

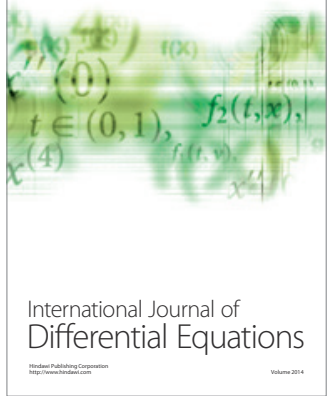
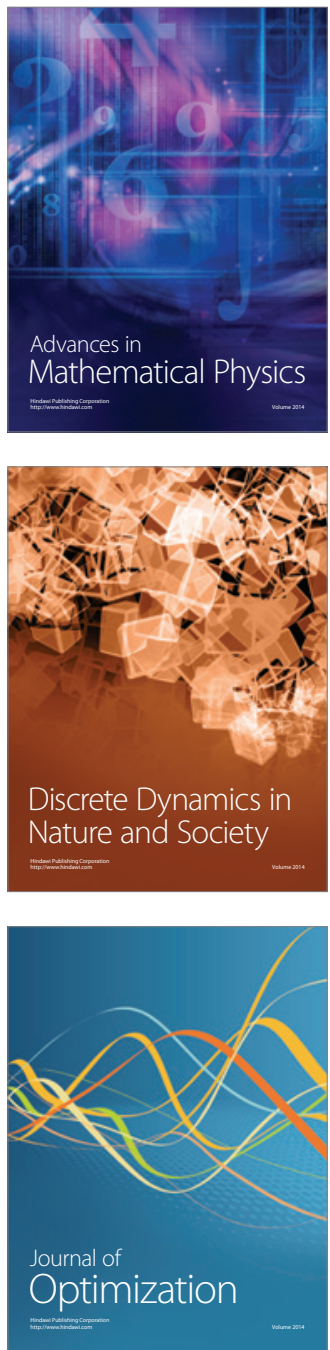